Monatsschrift f. Geburtshülfe u. Gynäkologie 1934;96:I-IV

\title{
Contents, Vol. 96, 1934
}

Inhaltsverzeichnis.

Originalarbeiten. Seite

Bublitseheoko, L. I., Der Abortus und die Sterilität 183

Bychowskaja, A. S., Zur Bewertung der Pyramidonprobe bei gestörter

extrauteriner Schwangerschaft 286

Ebergényi, Alexander, Uber eine interessante Zirkulationsstörung

der Nabelschnur als seltener Grand zum Abort

10

Eisenreich, Otto, Über Geburten bei Entwicklungsanomalien der

Genitalorgane 15

-, Uber seltenere Indikationen zur Unterbreehung der Schwangerschaft 5 d'Erchia, F., Beitrag zum Studium der hohen undfesten Vesiko-Vaginal-

fisteln mit besonderer Berücksichtigung der Therapie

-, Die Kolpozystotomie bei der Entfernung von Premdkörpern aus der

Harnblase 130

Fleckner, H., Zur Frage der Wachstumsstoffe im Menstrualblut ... 118 Frei, Jos., Erfahrungen

mit der Interposito uteri vesico-vaginalis nach

Schauta-Wertheim 135

Granzow, Joachim, Uber die Kraniotomie bei Kopflagen 247

Haupt, Walther, Zur geburtshilflichen Diagnose von Thorakopagen 148 Juzelevskij, A., siehe

Spoljanskij, G.

Kittner, Hans, Zur Behandlung der weiblichen Gonorrhoe unter be

sonderer Berücksichtigung der Flavadintherapie HI

Kolegaew, G. A., siehe Logwinsky, P. N.

Krupsky, A. J., Uber die zeitweilige chirurgische Unfruchtbarmachung

der Frau per vaginam. (Nach dem Verfahren des Verfassers.) ... 1 Kühbacher, F., Ein Beitrag zur Verfeinerung der Differentialdiagnose

der engen Becken; über die „Conjugata lateralis” 216

Legiehn, Herbert, siehe Schübel, Konrad.

Litwak, M. J., Beitrag zur Behandlung der Nachgeburtsperiode ... 24

Logwinsky, P. N., und G. A. Kolegaew, Die klinischen Erschei-

nungen von Endometritis und ihr Verhältnis zum pathologisch-histo-

logischen Bild 124

Ochssée, M. Ch. Boon von, siehe Tholen, M. H. G. A.

Rhemann, Franz, Hakenzange für Steißlage 297

Schübel, Konrad, Über die angebliche Potenzierung bei Kombination

von Wehenmitteln. Eine Entgegnung zum gleichen Thema von

Herbert Legiehn 270

Schulz, Herbert, Knochensystemstudien bei Hydrops foetus universalis 36

Sengupta, B. M., Zur Frage der Implantationsendometriose 262 
Sommer, Fritz, Akute Myositis haemorrhagica. Ein Fall einer postgrippösen Komplikation nach Sectio caesarea 200 Spiegler, Rudolf, Welche Bedeutung kommt der galvanischen Erregbarkeit bei der Erkennung der Eklampsie zu? $\quad 280$ Spoljanskij, G. und A.Juzelevskij,Der Hirndruckbei Enklampsieim Zusammenhang mit der Frage über die Atiologie der Krampfanfälle 190 Szüsz, Franz, Untersuchungen mit Bitterlingen zur Erkennung der

Schwangerschaft 292

JY Inhaltsverzeichnis.

S $\beta i t \beta$ Thi erf elder, Wolfgang, Die mikroskopisclie Senkungsbeobachtung

(Kriele) und ihre Verwertbarkeit zur Prognose und Difierentialprognose

genital bedingter septischer Infektionen 204

Tholen, M. H. G. A. und M. C. Boon von Ochssée, Abgeänderte

Kjellandzange 41

Unseld, E., Nierenaplasie und Doppelmißbildung des Uterus

Vogel, Herbert, Über die Normalhaltung des kindlichen K'opfes vor

seiner Fixierung auf dem mütterlichen Boden

Gre $z$ zgebiete der Geburtshilfe und Gynäkologie. Die Rõntgentherapie in der Gynäkologie vom

Juli 1932 bis Juli 1933. Von

Franz Wittenbeck, Erlangen 53

Die Radiutntherapie in der Gynäkologie. Ein Sammelbericht über ihre Ent-

wicklung in den letzten zwei Jahren. Von Friedrich Voltz, München 71 Berîcht über

innersekretorische Literatur (November 1932 bis Oktober 1933).

Von Karl Ehrhardt und Heinz Christian, Frankfurta. M. . . 156 Serologie des Karzinoms und der

Schwangerschaft (III). Von Erich

Tscherne, Graz 224

Narkose und Dämmerschlaf. (1933.) Von C. Schroeder, Würzburg. . . 301 Cholezystitis,

Cholelithiasis und Appendizitis. Von Eugen Kulka, Prag. 311

Sitzu $\pi$ gsberichte aus geburtshilflich-gynäkologischen Gesellschaften.

Vom 23. Deutschen Gynäkologenkongreß in Berlin am 11.-14. Oktober 1933.

Von G. H. Schneider, Brandenburg a. d. H 85

Gesellschaft für Geburtshilfe und Gynäkologie zu Berlin. Sitzungen vom

3. und 24. Februar $1933 \quad 94$

Sitzung vom 10. März $1933 \quad 237$

Gynäkologische Gesellscliaft in Breslau. Sitzung vom 7. November 1933169

Münchener gynäkologische Gesellschaft. Sitzung vom 18. Mai 1933 . . 171

Sitzung vom 13. Juli $1933 \quad 174$

Mittelrheinische Gesellschaft für Geburtshilfe und Gynäkologie. 88. Sitzung

vom 26. Juni 1932 in Frankfurt a. M 314

90. Sitzung vom 21. Mai 1932 in Frankfurt a. M

322

Geburtshilflich-gynäkologische Gesellschaft in Wien. Sitzung vom

14. November $1933 \quad 177$

Sitzungen vom 28. November und 12. Dezember 1932 und 16. Januar 1934

Buchbesprechungen 105, 179, 244, 346

Personalien und Tagesnachrichten $108,180, \quad 350$
A. Martin $\dagger$
109 
Max Walthard †

Otto Pankow $\uparrow$
106

347 